\title{
EFFECTIVE RESULTS FOR COMPLEX HYPERBOLIC MANIFOLDS
}

\author{
GABRIELE DI CERBO AND LUCA F. DI CERBO
}

\begin{abstract}
The goal of this paper is to study the geometry of cusped complex hyperbolic manifolds through their compactifications. We characterize toroidal compactifications with non-nef canonical divisor. We derive effective very ampleness results for toroidal compactifications of finite volume complex hyperbolic manifolds. We estimate the number of ends of such manifolds in terms of their volume. We give effective bounds on the number of complex hyperbolic manifolds with given upper bounds on the volume. Moreover, we give two sided bounds on their Picard numbers in terms of the volume and the number of cusps.
\end{abstract}

\section{Contents}

1. Introduction

1.1. Preliminaries

2. A gap theorem

3. Applications

3.1. Effective birationality

3.2. Bounds on the number of cusps

3.3. Bounds on the number of varieties 13

3.4. Bounds on the Picard numbers 15

References

\section{INTRODUCTION}

Let $\mathcal{H}^{n}$ be the complex hyperbolic space of dimension $n \geq 2$, that is, the Kähler space form with holomorphic sectional curvature -1 . Let $X^{o}$ be a complete noncompact complex hyperbolic manifold of finite volume. Such manifold is obtained as $X^{o}:=\mathcal{H}^{n} / \Gamma$ where $\Gamma$ is torsion-free lattice of $\mathrm{PU}(1, n)$ with parabolic elements which is non-uniform. Then $X^{o}$ has finitely many disjoint unbounded ends of finite volume, the cusps of $X^{\circ}$.

Baily-Borel BB66] and Siu-Yau SY82 proved that we can find a compactification $X^{*}$ of $X^{o}$ such that the complement of $X^{o}$ in $X^{*}$ consists of only finitely many (singular) points, called cusp points. Furthermore, under some mild assumptions on the lattice $\Gamma$, there exists a smooth variety $X$, which is a resolution of $X^{*}$, such that each exceptional divisor over a cusp point is a smooth abelian variety. For the precise requirements on $\Gamma$ we refer the interested reader to Section 1.1. Let us 
denote by $D=\coprod_{i} D_{i}$ the union of the exceptional divisors on $X$. We will refer to the pair $(X, D)$ as a toroidal compactification of $X^{o}$. The dimension of $X$ will always be denoted by $n$ and assumed to be greater or equal than two. Such toroidal compactifications were first constructed by Mumford et al. AMRT10, and by Mok Mok12], for more details see again Section 1.1 .

The goal of this work is to understand how the positivity properties of the logcanonical divisor $K_{X}+D$ determine the geometry of $X^{o}$ and $(X, D)$. The first technical result of this paper is that $K_{X}+D$ is not only big and nef but it is a limit of ample divisors of the form $K_{X}+\alpha D$ where $\alpha$ is a real number less than one. Moreover, there exists a uniform bound on the ampleness range independent of the dimension. Remarkably, the existence of such a uniform bound is crucial for all the geometric applications presented in this work.

Theorem 1.1. Let $(X, D)$ be a toroidal compactification. Then $K_{X}+\alpha D$ is ample for all $\alpha \in\left(\frac{1}{3}, 1\right)$.

The proof of Theorem 1.1 is based on the techniques developed by the authors in DiC12. We list here some of the main applications. We refer to Section 3 for details.

The first bi-product of the proof of Theorem 1.1 is a theorem characterizing toroidal compactifications with non-nef canonical divisor. Note that toroidal compactifications of ball quotients with non-nef canonical divisor do exist, see the paper by Hirzebruch [Hir84]. The surfaces constructed in [Hir84] are blow ups of a particular Abelian surface at certain configurations of points. These are the only currently known examples and unfortunately they are all in dimension two! We hope Theorem 1.2 below will help in the construction of higher dimensional examples and shed some light on the problem of determining how common or rare these examples are.

Theorem 1.2. Let $(X, D)$ be a toroidal compactification such that $K_{X}$ is not nef. Then $X$ is the blow-up of a smooth variety $Y$ along a smooth subvariety $Z$ of codimension 2 .

Theorem 1.2 follows from a classification result of extremal rays of toroidal compactifications. In particular, these varieties appear to be very simple from the minimal model point of view. This is quite unusual in higher dimension.

Next, Theorem 1.1 can successfully be applied to study the birational geometry of the log-canonical divisor of the pair $(X, D)$.

Theorem 1.3. Let $(X, D)$ be a toroidal compactification. Then for all $m \geq$ $(2 n+2)^{3}$, the map associated to $\left|m\left(K_{X}+D\right)\right|$ is a morphism and it defines an embedding of $X \backslash D$ into some projective space. Furthermore, this morphism maps all the different components of $D$ to distinct singular points.

Theorem 1.3 is an effective version of a recent result of Mok, see Main Theorem in Mok12]. Nevertheless, our proof relies on completely different techniques than Mok's. Note that when $(X, D)$ is a toroidal compactification associated to a neat 
arithmetic lattice in $\mathrm{PU}(1, n)$, Theorem 1.3 gives a very concrete realization of the classical Baily-Borel compactification $X^{*}$ as a projective algebraic variety.

Theorem 1.1 can also be applied to study the geometry of the smooth variety $X$ in the pair $(X, D)$.

Theorem 1.4. Let $(X, D)$ be a toroidal compactification. Then $X$ can be realized as a smooth subvariety of $\mathbb{P}^{2 n+1}$ such that its degree $d$ satisfies

$$
d \leq(2 n+2)^{3 n}\left(K_{X}+D\right)^{n} .
$$

A similar result is obtained by Hwang in Hwa05. The main advantage of our approach is that it provides bounds which are linear in $\left(K_{X}+D\right)^{n}$. The bounds obtained by Hwang are polynomial of degree $n+1$ in $\left(K_{X}+D\right)^{n}$.

Theorem 1.4 has several applications. For example, combining this result with classical Chow variety techniques, we can derive explicit bounds for the number of complex hyperbolic manifolds with given upper bounds on the volume. These results are effective versions of the classical Wang's finiteness theorem, see Wan72. For the numerical values of these bounds see Corollaries 3.14 and 3.17

As a final application of Theorem 1.1, we show how to bound the number of cusps of a finite volume complex hyperbolic manifold in terms of its volume.

Theorem 1.5. Let $(X, D)$ be a toroidal compactification. Let $q$ be the number of cusps of $X^{\circ}$. Then

$$
q \leq\left(\frac{3}{2}\right)^{n}\left(K_{X}+D\right)^{n}
$$

The problem of bounding the number of ends in pinched negatively curved finite volume manifolds is a long standing problem in differential geometry. For complex hyperbolic manifolds, the classical approach is via techniques coming from geometric topology, see Par98 and the bibliography therein. More recently, Hwang in [Hwa04] was able to improve Parker's result by using the Hirzebruch-Mumford proportionality principle Mum77. The bound we present here in Theorem 1.5 is better than the one obtained by Parker but worse than Hwang's for $n \geq 4$. We decided to include this result here mainly for two reasons. First, it appears to be the best currently known bound for surfaces and threefolds. Moreover, the proof of Theorem 1.5 is completely elementary and it follows easily from Theorem 1.1 . Second, the reasoning given in the proof of Theorem 1.5 is in principle applicable in a much general setting, while both the approaches of Parker and Hwang use in an essential way the special properties of complex hyperbolic manifolds.

In Section 3.2, we describe an alternative approach to the problem of bounding the cusps, see Proposition 3.12 This approach does not rely on Theorem 1.1 but rather on an old result of Matsusaka. The strategy of the proof can again be in principle applied to a much more general setting than ball quotients, e.g., compactifications of finite volume Kähler manifolds with pinched variable negative curvature.

Finally, in Section 3.4 we show how the techniques developed in this paper can be successfully applied to bound from above the Picard numbers of toroidal 
compactifications in terms of their volume. Moreover, we give a lower bound on the Picard number in terms of the number of cusps. For the details see Theorems 3.18 and 3.19

1.1. Preliminaries. The theory of compactifications of locally symmetric varieties has been extensively studied, see for example [BJ06]. For technical reasons this theory is mainly developed for quotients of symmetric spaces by arithmetic subgroups. In most cases this is not a serious issue since the work of Margulis Mar84 implies that lattices in any semi-simple Lie group of real rank bigger or equal than two are arithmetic subgroups. Nevertheless, this theorem does not cover the case of lattices in the complex hyperbolic space $\mathcal{H}^{n}$ which are the main object of study in this paper. Note that non-arithmetic lattices in $\mathrm{PU}(1, n)$ were constructed by Mostow and Deligne-Mostow; see [DM93] and the extensive bibliography there.

It would then be desirable to develop a theory of compactifications of finite volume complex hyperbolic manifolds which does not rely on the arithmeticity of the defining torsion free lattices. Fortunately, this problem was addressed by many mathematicians from several different point of views. A compactification of finite volume complex hyperbolic manifolds as a complex spaces with isolated normal singularities was obtained by Siu and Yau in [SY82]. This compactification may be regarded as a generalization of the Baily-Borel compactification defined for arithmetic lattices in $\mathcal{H}^{n}$. A toroidal compactification for finite volume complex hyperbolic manifolds was described by Hummel and Schroeder in [HS96]. In many cases these compactifications provide explicit resolution of singularities of the Siu-Yau compactifications. More recently Mok Mok12 gave a lucid and detailed description of these compactifications and described many of their remarkable features.

The compactifications studied in this work are the ones described by HummelSchroeder and Mok. Thus, we do not require that lattice $\Gamma$ to be arithmetic. On the other hand, we require that all the parabolic isometries of $\Gamma$ are unipotent, in other words we require that they act by translations on their invariant horospheres. We impose this condition in order to obtain smooth toroidal compactifications. We would like to point out that this technicality is hidden in the construction described by Mok while it is explicitly discussed in the work of Hummel-Scroeder. Let us describe in more details this technical point. Recall that given a non-uniform torsion-free lattice $\Gamma \leq \mathrm{PU}(1, n)$, the finite volume complex hyperbolic manifold $\mathcal{H}^{n} / \Gamma$ has finitely many cusps $C_{1}, \ldots, C_{m}$ which are in one to one correspondence with the maximal parabolic subgroups of $\Gamma$, see [Ebe96] for more details. Given a cusp $C_{i}$, denote by $\Gamma_{i} \leq \Gamma$ the associated maximal parabolic subgroup and by $\mathrm{HB}_{i}$ the horoball stabilized by $\Gamma_{i}$. After choosing an Iwasawa decomposition [Ebe96] for $\mathrm{PU}(1, n)$, we can identify $\partial \mathrm{HB}$ with a Heisenberg type Lie group $N_{i}$ diffeomorphic to $\mathbb{C}^{n-1} \times \mathbb{R}$. Thus, the center $Z_{i}$ of $N_{i}$ is $Z_{i}=\left[N_{i}, N_{i}\right]$ and it is isomorphic to $\mathbb{R}$. Furthermore, the simply connected Lie group $N_{i}$ comes equipped with a natural left invariant metric and then we can consider $\Gamma_{i}$ as a lattice in $\operatorname{Iso}\left(N_{i}\right)$. The isometry group of $N$ is isomorphic to the semi-direct product $\operatorname{Iso}\left(N_{i}\right)=N_{i} \rtimes$ $U(n-1)$. We then say that $\phi \in \Gamma_{i}$ is unipotent if it is a translation in $\operatorname{Iso}\left(N_{i}\right)$. 
Now, the construction described by Hummel-Scroeder and Mok produces a smooth compactification if the quotients $\Gamma_{i} / \Gamma_{i} \cap Z_{i}$ are torsion free for all $i$. This is the case if all the parabolic isometries of $\Gamma$ are unipotent. In the classical arithmetic case this requirement is usually satisfied by requiring the lattice $\Gamma$ to be neat, see [BB66] and BJ06] for more details. Let us note that given any non-uniform lattice $\Gamma \in \mathrm{PU}(1, n)$, there always exists a finite index subgroup whose all parabolic isometries are unipotent, in the arithmetic case see [AMRT10] while for the general non-arithmetic case we refer to Hum98].

Acknowledgements. The first named author would like to express his gratitude to Professor János Kollár for his constant support and for valuable discussions on the results contained in this work. Both authors are grateful to Cinzia Casagrande for pointing out the reference Wis91] and for generously sharing her knowledge. This reference was crucial in obtaining a bound which is uniform in dimension in Theorem 1.1. We thank the organizers of the conference "Algebraic \& Hyperbolic Geometry - New Connections" where a preliminary version of this work was presented and were part of the later improvements were conceived.

\section{A GAP THEOREM}

In this section we prove Theorem 1.1 which is the main technical result of the paper. The proof of Theorem 1.1 is based on the following result contained in DiC12, see Theorem 4.15.

Theorem 2.1. Let $X$ be a smooth projective variety and let $D$ be a reduced effective divisor with simple normal crossing support such that $K_{X}+D$ is big and nef. Then $K_{X}+\alpha D$ is ample for $\alpha \in\left(\frac{n+1}{n+2}, 1\right)$ if and only if there are no irreducible curves $C$ such that $\left(K_{X}+D\right) \cdot C=0$ and $K_{X} \cdot C \leq 0$.

The key ingredient in the proof of Theorem 2.1 is the Cone Theorem. In particular the fact that $\alpha \in\left(\frac{n+1}{n+2}, 1\right)$ is obtained using the bound on the length of extremal rays given in the Cone Theorem. Let us recall the definition of an extremal ray. We refer to [Kol96] and KM98 for more details.

Definition 2.2. Let $N \subset \mathbb{R}^{m}$ be a cone. A subcone $M \subset N$ is called extremal if $u, v \in N, u+v \in M$ imply that $u, v \in M$. A 1-dimensional extremal subcone is called an extremal ray.

In this paper we will consider only extremal rays $R$ of the cone of effective 1cycles $\overline{N E}(X)$ such that $K_{X} \cdot Z<0$ for any effective 1 -cycle $Z$ in $R$. For such extremal rays we define the length of $R$ as

$$
l(R):=\min \left\{-K_{X} \cdot C \mid C \text { is a rational curve with numerical class in } R\right\} .
$$

The length of extremal rays in $\overline{N E}(X)$ has been extensively studied. The Cone Theorem gives that $l(R) \leq n+1$. On the other hand, there are many classification results regarding varieties with extremal rays of maximal length. We will prove a strong bound on the length of extremal rays of toroidal compactifications. 
Theorem 2.3. Let $(X, D)$ be a toroidal compactification of dimension $n$. Then $l(R) \leq 1$ for any extremal ray $R$.

We will prove Theorem 2.3 in few steps. The first and key result is the following.

Lemma 2.4. Let $(X, D)$ be a toroidal compactification. Let $R$ be an extremal ray of $X$ and let $F$ be an irreducible component of a non-trivial fiber of the contraction of $R$. Then $\operatorname{dim}(F) \leq 1$.

Proof. Let $C$ be a curve in $F$. Since $C$ is contracted by the contraction of $R$, the Contraction Theorem, see [KM98, gives that $C \in[R]$. Since $R$ is $K_{X}$-negative and $K_{X}+D$ is nef, we have that $D \cdot C>0$. On the other hand, if $C \subseteq D_{i}$, then $D \cdot C<0$ because the normal bundle of $D_{i}$ is anti-ample. In particular, $\operatorname{dim}(D \cap F)=0$ which implies $\operatorname{dim}(F) \leq 1$.

The dimension of the fibers of the contraction controls the length of the corresponding extremal ray. Recall the following result in [Wis91.

Theorem 2.5 (Wiśniewski). If $F$ is a nontrivial fiber of a contraction of $R$ then

$$
\operatorname{dim}(F) \geq l(R)-1 \text {. }
$$

Combining Theorem 2.5 and Lemma 2.4 we obtain the following.

Corollary 2.6. Let $(X, D)$ be a toroidal compactification and let $R$ be an extremal ray of $X$. Then $l(R) \leq 2$.

In order to prove Theorem 2.3 , it suffices to prove that the case $l(R)=2$ and $\operatorname{dim}(F) \leq 1$ does not occur. Such extremal contractions have been classified by Wiśniewski Wis91.

Theorem 2.7 (Wiśniewski). Let $X$ be a smooth variety. Let $\phi: X \rightarrow Y$ be the contraction of an extremal ray $R$ of $X$ such that $\operatorname{dim}(F) \leq 1$. Then $Y$ is smooth and either

(1) $\phi: X \rightarrow Y$ is a conic bundle, or

(2) $\phi: X \rightarrow Y$ is the blow-up of the variety $Y$ along a smooth subvariety $Z$ of codimension 2.

Our goal is to prove that toroidal compactifications do not admit a conic bundle structure. This concludes the proof of Theorem 2.3 .

Proof of Theorem 2.3. By Lemma 2.4 and Theorem 2.7 we need to show that $\phi$ : $X \rightarrow Y$ cannot be a conic bundle. We first deal with the case $\operatorname{dim}(X)=2$. In this case $l(R)=2=\operatorname{dim}(X)$ and by Proposition 2.4.2 in [Wis89], we obtain that the Picard number of $X$, say $\rho(X)$, is equal to two. Since $D$ is an elliptic curve, we have that $\phi$ restricted to $D$ is a surjective morphism and in particular $g(Y) \leq 1$. Since $\rho(X)=2$, the ruled surface $X$ must be minimal. If $Y$ is a rational curve then $X$ is a Hirzebruch surface. On a Hirzebruch surface the only curve with negative self intersection is the zero section, which is a rational curve, so it cannot be $D$.

We can then assume that $Y$ is an elliptic curve. Since $X$ is minimal, we know that $X=\mathbb{P}(\mathcal{E})$ where $\mathcal{E}$ is a normalized rank 2 vector bundle over $Y$. Following 
Proposition V.2.8 in Har77, we can normalize $\mathcal{E}$ so that the integer $e=-\operatorname{deg}(\mathcal{E})$ is an invariant on $X$. Recall that, under this normalization, the canonical section of $X$, say $C_{0}$, satisfies $C_{0}^{2}=-e$. Since $Y$ is elliptic, by a theorem of Atiyah we know that $e \geq-1$, see Theorem V.2.15 in Har77. If $e \leq 0$ then $-K_{X}$ is nef and in particular by adjunction $D^{2} \geq 0$. We then have $e \geq 1$. By Proposition V.2.20 in Har77, any irreducible curve $Y \neq C_{0}, F$ must be numerically equivalent to

$$
Y \equiv a C_{0}+b F
$$

where $F$ is a fiber of the ruling and $a>0, b \geq a e$. Thus

$$
Y^{2}=-a^{2} e+2 a b \geq a^{2} e \geq 0
$$

which then shows that the only curve with negative self-intersection is the zero section. This implies that $D$ is the zero section. We can then find a $\mathbb{P}^{1}$ with just one puncture in $X \backslash D$. Since curvature can only decrease along complex submanifolds of a negatively curved Kähler manifold, we obtain a contradiction.

Now suppose that $\operatorname{dim}(X) \geq 3$ and $\phi$ is a conic bundle. We will work on the Baily-Borel compactification $X^{*}$. Recall that $X^{*}$ is obtained from $X$ contracting the components of the divisor $D$. In particular, the fibers of $\phi$ define a family of rational curves on $X^{*}$. Let $F$ be a fiber of $\phi$. First, assume that every fiber of $\phi$ is a smooth conic. Since $D \cdot F \geq 3$, the family of rational curves on $X^{*}$ has at least three fixed points. Recall that bend and break, see Lemma 1.9 in [KM98, implies that if a family of rational curves moves with at least two fixed points then it must split, i.e. it contains a reducible curve. Applying bend and break to our situation, we obtain that the family of rational curves on $X^{*}$ contains a reducible curve which corresponds to a reducible fiber of $\phi$. This contradicts our assumption on the smoothness of the fibers.

In general, $\phi$ has reducible fibers. Since the discriminant locus of a conic bundle is a divisor on the base, the reducible fibers form a new family of rational curves on $X^{*}$ of positive dimension because $\operatorname{dim}(X) \geq 3$. The family formed by the irreducible components has at least two fixed points and applying again bend and break, we obtain a new splitting. In particular, we have a fiber with three components. This is a contradiction because every fiber of $\phi$ is isomorphic to a conic in $\mathbb{P}^{2}$.

Theorem 2.3 implies an interesting structure result on toroidal compactifications with non-nef canonical divisor.

Corollary 2.8 (Theorem 1.2). Let $(X, D)$ be a toroidal compactification such that $K_{X}$ is not nef. Then $X$ is the blow-up of a smooth variety $Y$ along a smooth subvariety $Z$ of codimension 2 .

We can now prove Theorem 1.1 .

Proof of Theorem 1.1. First, we would like to show that $K_{X}+D$ is big and nef. Recall that $\mathcal{H}^{n}$ is the negatively curved complex space form. Thus, any manifold $X^{o}=\mathcal{H}^{n} / \Gamma$, with $\Gamma$ non-uniform, is equipped with a standard negatively curved Kähler-Einstein metric of finite volume which we denote by $\hat{\omega}$. By Théorème 1.1 in 
Sib85, such a metric $\hat{\omega}$ on $X^{o}$ can be regarded as a closed positive current on $X$. Moreover, it in not difficult to see that $\hat{\omega}$ is in the cohomology class of $K_{X}+D$. More precisely, let $D_{i}$ be the irreducible components of $D$ and let $s_{i} \in \mathcal{O}_{X}\left(D_{i}\right)$ be the defining sections. By appropriately choosing Hermitian metrics $\|\cdot\|_{i}$ on $\mathcal{O}_{X}\left(D_{i}\right)$, the volume form associated to $\hat{\omega}$ is then of the form

$$
\Psi=\frac{\Omega}{\prod_{i}\left\|s_{i}\right\|^{2}\left(-\log \left\|s_{i}\right\|^{2}\right)^{n+1}}
$$

for some globally defined volume form $\Omega$ on $X$. Thus, the Poincaré-Lelong formula combined with the fact that $\hat{\omega}$ is Kähler-Einstein gives that $\hat{\omega} \in\left[K_{X}+D\right]$. Next, let us observe that $\omega_{P} \geq \hat{\omega}$ for some Kähler current $\omega_{P}$ on $X$ with Poincaré type singularities along $D$. We then have that $\hat{\omega}$ is a closed positive current with zero Lelong numbers on $X$. Moreover, since $\hat{\omega}$ is a regular Kähler metric on $X^{o}$, it is strictly positive at any point $p \in X \backslash D$. By using a regularization argument based on results of Demailly Dem92, we conclude that $K_{X}+D$ is big and nef, see also Theorem 1.3 in [JS93. We can actually conclude more. In fact, we must have $\left(K_{X}+D\right)^{\operatorname{dim}(Z)} \cdot Z>0$ for any subvariety $Z$ not contained in $D$.

Finally, we want to show that if $C$ is a curve such that $\left(K_{X}+D\right) \cdot C=0$ then $K_{X} \cdot C>0$. By the discussion above we know that $C$ must be contained in $D$. Then

$$
K_{X} \cdot C=-D \cdot C>0
$$

since the normal bundle of $D$ in $X$ is anti-ample, see Theorem 1 in Mok12. In particular Theorem 2.1 implies that $K_{X}+\alpha D$ is ample for all values of $\alpha$ close enough to one. Let us denote by $\left\{C_{i}\right\}$ the generators of the extremal rays in $X$. Each $C_{i}$ is a smooth rational curve and $D \cdot C_{i} \geq 3$ for all $i$. If otherwise there would exists a $\mathbb{P}^{1}$ with less than three punctures in $X \backslash D$ which is clearly impossible. We then conclude that $\left(K_{X}+D\right) \cdot C_{i} \geq 2$ for all $i$. Following the proof of Theorem 2.1 in $\mathrm{DiC12}$ and using the bound given by Theorem 2.3, it follows that the $K_{X}+\alpha D$ is ample for all $\alpha \in\left(\frac{1}{3}, 1\right)$.

Remark 2.9. Theorem 1.1 is geometrically sharp as it relates the existence of a "gap", in the ampleness range of $K_{X}+\alpha D$, only to the number of times a smooth rational curve in the ambient space $X$ intersects the boundary D. Elementary hyperbolic geometry tells us that this number must be greater or equal than three, and then the bound in Theorem 1.1. Unfortunately, in all of the examples constructed by Hirzebruch in [Hir84, the rational curves intersect the boundary in four points. Thus, Theorem 1.1 is currently not numerically sharp. It would be extremely interesting to construct examples of toroidal compactifications with non-nef canonical divisor, having a rational curve that intersects the boundary in just three points.

It seems a difficult problem to understand which varieties arise as toroidal compactifications of hyperbolic manifolds. The above theorem gives a first step toward a possible solution of the problem. If $n \geq 3$, it follows also from Lefschetz hyperplane theorem. 
Corollary 2.10. There are no toroidal compactification $(X, D)$ with $X$ a smooth Fano variety.

Proof. Suppose $(X, D)$ is a toroidal compactification with $-K_{X}$ ample. Because of Theorem 1.1, for all $\alpha$ close to one, we know $K_{X}+\alpha D$ is ample. By Corollary 4.18 in [DiC12, it follows that $K_{X}+D$ is strictly nef. On the other hand, we must have $\left(K_{X}+D\right)_{\left.\right|_{D}}=\mathcal{O}_{D}$. This is a contradiction.

Question 2.11. It is interesting to ask whether there exists a smooth toroidal compactification of a ball quotient with negative Kodaira dimension.

\section{Applichtions}

In this section, we give the proofs of Theorems $1.3,1.4$ and 1.5 stated in the Introduction.

3.1. Effective birationality. Let us start by studying the birational properties of the divisor $K_{X}+D$. First, we prove that the map associated to $\left|m\left(K_{X}+D\right)\right|$ maps the components of $D$ to distinct points for any $m \geq 2$.

Proposition 3.1. Let $(X, D)$ be a toroidal compactification. Then for any $i$ there exists a section $\sigma_{i}$ of $H^{0}\left(X, \mathcal{O}_{X}\left(2\left(K_{X}+D\right)\right)\right)$ such that $\left.\sigma_{i}\right|_{D_{i}} \neq 0$ and $\left.\sigma_{i}\right|_{D_{j}}=0$ for all $j \neq i$.

Proof. Write $D=\sum_{i=1}^{q} D_{i}$ and recall that each component is an abelian variety and they are all disjoint. Consider the following exact sequence

$$
0 \rightarrow \mathcal{O}_{X}\left(2 K_{X}+D\right) \stackrel{\cdot D}{\rightarrow} \mathcal{O}_{X}\left(2\left(K_{X}+D\right)\right) \rightarrow \mathcal{O}_{D} \rightarrow 0
$$

By Kawamata-Viehweg's vanishing we have that $H^{1}\left(X, \mathcal{O}_{X}\left(2 K_{X}+D\right)\right)=0$. Thus, taking the long exact sequence in cohomology, we get the following surjective map

$$
H^{0}\left(X, \mathcal{O}_{X}\left(2\left(K_{X}+D\right)\right)\right) \rightarrow \bigoplus_{i=1}^{q} H^{0}\left(D_{i}, \mathcal{O}_{D_{i}}\right)
$$

The next step is to understand what happens outside the boundary divisor. Let us start by deriving a lower bound on the top self-intersection of $K_{X}+D$.

Lemma 3.2. Let $(X, D)$ be a toroidal compactification. Then

$$
\left(K_{X}+D\right)^{n} \geq(n+1)^{n-1} .
$$

Proof. Since the Kähler-Einstein current $\hat{\omega}$ can be multiplied by itself, the top selfintersection of $L:=K_{X}+D$ can be expressed in terms of the Riemannian volume of $X^{o}$. More precisely, by normalizing the holomorphic sectional curvature to be -1 we have

$$
\operatorname{Vol}(X)=\frac{(4 \pi)^{n}}{n !(n+1)^{n}} L^{n}
$$


On the other hand, Gromov-Harder's generalization of Gauss-Bonnet Gro82 implies that

$$
\operatorname{Vol}(X) \geq \frac{(4 \pi)^{n}}{(n+1) !}
$$

Combining the two formulas above we get the result.

These considerations and a theorem of Kollár in Kol97] imply the following.

Corollary 3.3. Let $(X, D)$ be a toroidal compactification. Then $m\left(K_{X}+D\right)$ is base point free for any $m \geq\left(\begin{array}{l}n \\ 2\end{array}\right)+1$.

Proof. By Theorem 1.1, for any subvariety $Z$ not contained in $D$ we have $\left(K_{X}+\right.$ $D)^{\operatorname{dim}(Z)} \cdot Z>0$. Because of Theorem 5.8 in [Kol97], we know that $m\left(K_{X}+D\right)$ is free at all points outside $D$ for any $m \geq\left(\begin{array}{l}n \\ 2\end{array}\right)+1$. Moreover, by Lemma 3.1 we know already that $2\left(K_{X}+D\right)$ is free on the divisor $D$.

Similarly, we can study separation of points.

Corollary 3.4. Let $(X, D)$ be a toroidal compactification. Then $m\left(K_{X}+D\right)$ separates any two points in $X \backslash D$ for any $m \geq\left(\begin{array}{l}n \\ 2\end{array}\right)+2$.

Proof. Combine Theorem 5.9 in [Kol97] and Lemma 3.2.

Since $X \backslash D$ is open in $X$, Corollary 3.4 implies that $\left|m\left(K_{X}+D\right)\right|$ defines a birational map for any $m \geq\left(\begin{array}{l}n \\ 2\end{array}\right)+2$. These simple results can be already used to slightly improve all the bounds in Hwa05]. Nevertheless, the approach we follow here is quite different. Note that Corollary 3.4 deals only with separation of points. Thus, if we want to prove that $m\left(K_{X}+D\right)$ defines an embedding outside $D$ we need something more. Again the key for us is Theorem 1.1, while the approach described in Hwa05] relies on Seshadri constants type arguments. Let us start by recalling the following definition.

Definition 3.5. Let $X$ be a smooth projective variety and let $D$ be an effective divisor. We say that a divisor $L$ is very ample modulo $D$ if the map $\phi_{L}: X \rightarrow$ $\mathbb{P}\left(H^{0}\left(X, \mathcal{O}_{X}(L)\right)\right)$ defines an embedding of $X \backslash D$.

We can now derive an effective result on very ampleness modulo $D$ of $K_{X}+D$. In order to keep the final statement simple, some of the constants used in the proof are not optimal.

Theorem 3.6. Let $(X, D)$ be a toroidal compactification of dimension $n$. Then $m\left(K_{X}+D\right)$ is very ample modulo $D$ for any $m \geq 2(n+1)^{3}$.

Proof. Theorem 1.1 implies that $K_{X}+\frac{1}{2} D$ is ample. Then $H:=2 K_{X}+D$ is an ample integral divisor. By a theorem of Angehrn-Siu we have that $K_{X}+m H$ is ample and base point free for any $m>\left(\begin{array}{c}n+1 \\ 2\end{array}\right)$, see [AS95] or Kol97] and Laz04b] for an algebraic proof. In particular $B:=K_{X}+\left(n^{2}+1\right) H$ is ample and base point free. A corollary of Castelnuovo-Mumford regularity, see Example 1.8.23 in 
Laz04a, gives that $K_{X}+(n+2) B$ is very ample. All together we have that the divisor

$$
\left(2\left(n^{2}+1\right)(n+2)+n+3\right) K_{X}+\left(n^{2}+1\right)(n+2) D
$$

is very ample. Let $M:=\left(2\left(n^{2}+1\right)(n+2)+n+3\right)$. Adding the right positive multiple of $D$ we get the following injective map

$$
H^{0}\left(X, \mathcal{O}_{X}\left(K_{X}+(n+2) B\right)\right) \hookrightarrow H^{0}\left(X, \mathcal{O}_{X}\left(M\left(K_{X}+D\right)\right)\right) .
$$

This implies that $m\left(K_{X}+D\right)$ is very ample modulo $D$ for any $m \geq M$. Since $2(n+1)^{3} \geq M$ we get the statement of the theorem.

Theorem 1.3 stated in the introduction is now just a combination of Theorem 3.6 and Proposition 3.1 .

Let us conclude this section by discussing the connections between Fujita's conjecture and Theorem 3.6 . First, recall that if $\operatorname{dim}(X) \leq 4$ then the base point free part of Fujita's conjecture is known to be true thanks to the work of Kawamata Kaw97. We can then improve the bound in Theorem 3.6 for low dimensional varieties.

Corollary 3.7. Let $(X, D)$ be a toroidal compactification of dimension $n \leq 4$. Then $m\left(K_{X}+D\right)$ is very ample modulo $D$ for any $m \geq 2(n+2)^{2}$.

Proof. It follows along the same lines of Theorem 3.6. Instead of using AngehrnSiu's theorem, we use Kawamata's result on Fujita's conjecture. The rest remains unchanged.

It is interesting to note that using a theorem of G. Heier in Hei02, we can get a bound of order $n^{7 / 3}$ in Theorem 3.6 . Of course an even better bound would be obtained using the very ampleness part of Fujita's conjecture. More precisely, assuming the conjecture of Fujita to be true, the proof of Theorem 3.6 easily gives that $m\left(K_{X}+D\right)$ is very ample modulo $D$ for any $m \geq 2(n+2)$.

3.2. Bounds on the number of cusps. In this section, we show how to explicitly estimate the number of cusps of $X^{o}$ in terms of the intersection number $\left(K_{X}+D\right)^{n}$. We present two different approaches. The first one is a direct corollary of Theorem 1.1. while the second bound follows easily from a result of Matsusaka. Moreover, it gives the best currently known bound for threefolds.

Recall that $X^{o}$ has finitely many ends which correspond to the cusp points of $X^{*}$. Each cusp point gives rise to one component of the boundary divisor $D$ in $X$. So bounding the number of cusps is equivalent to bounding the number of components of $D$.

We can now prove Theorem 1.5 stated in the Introduction.

Theorem 3.8 (Theorem 1.5). Let $(X, D)$ be a toroidal compactification. Let $q$ be the number of components of $D$. Then

$$
q \leq\left(\frac{3}{2}\right)^{n}\left(K_{X}+D\right)^{n}
$$


Proof. Once we have Theorem 1.1, the above result follows from a quite straightforward computation. Let $L:=K_{X}+D$. Because of Theorem 1.1, we know that $3 L-2 D$ is nef and $2 L-D$ is ample. Then

$$
q \leq D \cdot(2 L-D)^{n-1}=(-1)^{n-1} D^{n} .
$$

Since $3 L-2 D$ is nef we get that

$$
(3 L-2 D)^{n}=3^{n} L^{n}+(-1)^{n} 2^{n} D^{n} \geq 0 .
$$

Combining the two inequalities we get the result.

Note that we can also a bound on the top self-intersection of $D$ in terms of $\left(K_{X}+D\right)^{n}$ only. Let us summarize this fact in the form of a corollary as it will be used in Section 3.3

Corollary 3.9. Let $(X, D)$ be a toroidal compactification. Then

$$
(-1)^{n-1} D^{n} \leq\left(\frac{3}{2}\right)^{n}\left(K_{X}+D\right)^{n} .
$$

We now present an estimate on the number of cusps which does not rely on Theorem 1.1. The key point is the following corollary of Proposition 3.1.

Corollary 3.10. Let $(X, D)$ be a toroidal compactification. Let $q$ be the number of components of $D$. Then

$$
h^{0}\left(X, \mathcal{O}_{X}\left(2\left(K_{X}+D\right)\right)\right) \geq q
$$

In particular, in order to bound the number of cusps, it is enough to bound $h^{0}\left(X, \mathcal{O}_{X}\left(2\left(K_{X}+D\right)\right)\right.$. This can be done using a result of Matsusaka. For an outline of the proof we refer to VI.2.15.8.7 page 301 in Kol96].

Theorem 3.11 (Matsusaka). Let $H$ be a big and nef divisor on a smooth variety $X$ of dimension $n$. Then for any $m \geq 1$ we have

$$
h^{0}\left(X, \mathcal{O}_{X}(m H)\right) \leq m^{n} H^{n}+n .
$$

Combining these two results we get the following.

Proposition 3.12. Let $(X, D)$ be a toroidal compactification. Let $q$ be the number of components of $D$. Then

$$
q \leq\left(2^{n}+1\right)\left(K_{X}+D\right)^{n}
$$

Proof. By Lemma 3.2, we have that $\left(K_{X}+D\right)^{n} \geq n$.

It is interesting to note how the bounds given in Theorem 1.5 and Proposition 3.12 rely on few basic geometric properties of the pair $(X, D)$. Roughly speaking, they depend on the fact that $L=K_{X}+D$ is big, nef and such that $L_{\left.\right|_{D}}=\mathcal{O}_{D}$. None of the other special features of toroidal compactifications of ball quotients is used. Therefore, these techniques can be successfully applied to study complete finite volume Kähler manifolds with pinched negative sectional curvature which are not locally symmetric. These results will appear elsewhere. 
Finally, despite their simple nature, the bounds given in Theorem 1.5 and Proposition 3.12 are the best currently known bounds for surfaces and threefolds, compare with $\mathrm{Par98}$ and $\mathrm{Hwa04}$.

3.3. Bounds on the number of varieties. In this section we study the problem of finding effective bounds on the number of complex hyperbolic manifolds with bounded volume. The main issue is to find effective embedding results for toroirodal compactifications. This was successfully done in Theorem 3.6 and we investigate its consequences here. The key result of this section is Theorem 1.4 stated in the Introduction, which we restate for the convenience of the reader.

Theorem 3.13 (Theorem 1.4). Let $(X, D)$ be a toroidal compactification. Then $X$ can be realized as a smooth subvariety of $\mathbb{P}^{2 n+1}$ such that its degree $d$ satisfies

$$
d \leq(2 n+2)^{3 n}\left(K_{X}+D\right)^{n} .
$$

Proof. Let $L:=K_{X}+D$. By Theorem 3.6. we know that $2(n+1)^{3} L-k D$ is very ample, for some explicit positive integer $k$. Then $2(n+1)^{3} L-k D$ defines an embedding of $X$ into some projective space with degree $d \leq(2(n+1))^{3 n} L^{n}$. By general projection, we obtain that $X$ sits inside $\mathbb{P}^{2 n+1}$.

As pointed out in the Introduction, Hwang proved a similar result in Hwa05. While our bound is linear in $\left(K_{X}+D\right)^{n}$, he derived a polynomial bound in $\left(K_{X}+\right.$ $D)^{n}$ of degree $n+1$. Once we have Theorem 3.13 . counting the number of complex hyperbolic manifolds is reduced to standard techniques on the complexity of Chow varieties. Let us briefly explain this point. Associated to a hyperbolic manifold, we have a toroidal compactification $(X, D)$. Therefore, it is enough to count such pairs. Fujiki in Fuj92 proved that a toroidal compactification $(X, D)$ is infinitesimally rigid under deformations of the pair. This implies that the number of toroidal compactifications is bounded by the number of components of a suitable Chow variety, see Corollary 3.17. Moreover, Hwang pointed out that Fujiki's theorem implies something more. If $n \geq 3, X$ itself is rigid under deformations and the same method applies, see Proposition 4.2 in [Hwa05].

We start by counting the varieties which arise as toroidal compactifications of complex hyperbolic manifolds with bounded volume. In particular, in the next statement we forget about the extra structure coming from the boundary divisor.

Fix two positive integers $d$ and $m>n$. We denote by $\operatorname{Chow}_{m}(n, d)$ the Chow variety of $n$-dimensional irreducible smooth subvarieties of $\mathbb{P}^{m}$ of degree $d$.

Corollary 3.14. Fix two positive integers $n \geq 3$ and $V$. Then the number of varieties $X$ arising as toroidal compactifications $(X, D)$ with $\operatorname{dim}(X)=n$ and $\left(K_{X}+D\right)^{n} \leq V$ is bounded by

$$
\sum_{d=1}^{d_{0}}\left(\begin{array}{c}
(2 n+2) d \\
2 n+1
\end{array}\right)^{(2 n+2) d\left(\begin{array}{c}
d+n-1 \\
n
\end{array}\right)+(2 n+2)\left(\begin{array}{c}
d+n-1 \\
n-1
\end{array}\right)},
$$

where $d_{0}=(2 n+2)^{3 n} V$. 
Proof. Let $(X, D)$ be a toroidal compactification as in the statement. By Theorem 3.13. $X$ can be embedded in $\mathbb{P}^{2 n+1}$ as a smooth subvarieties of degree $d \leq d_{0}$. Proposition 4.2 in Hwa05 implies that $X$ is rigid under deformation. In particular, the number of such toroidal compactifications is bounded by the number of components of the Chow variety $\operatorname{Chow}_{2 n+1}\left(n, d_{0}\right)$. A straightforward application of I.3.28.9 in [Kol96] gives the result.

Remark 3.15. Independently of the value of $d_{0}$, the bound on the number of components of the Chow variety is of the form

$$
\left(a_{n} d_{0}\right)^{\left(b_{n} d_{0}\right)^{n+1}}
$$

for some function $a_{n}$ and $b_{n}$ which depend only on $n$. Therefore, the bound in Corollary 3.14, expressed just in terms of the volume, is of the form

$$
V^{V^{n+1}} \text {. }
$$

We can now give effective estimates on the number of toroidal compactifications $(X, D)$ with bounded $\left(K_{X}+D\right)^{n}$, counted as pairs. This corresponds exactly to bounding the number of complex hyperbolic manifolds with bounded volume. Unfortunately the bounds are slightly worst than the ones in Corollary 3.14

We need to study a different Chow variety which takes into account the boundary divisor. Given positive integers $q, m, n, n_{1}, \ldots, n_{q}, d, d_{1}, \ldots, d_{q}$ we define the Chow variety $\operatorname{Chow}_{m}\left(n, d ; n_{1}, \ldots, n_{q} ; d_{1}, \ldots, d_{q}\right)$ to be the closed subvariety of

$$
\operatorname{Chow}_{m}(n, d) \times \operatorname{Chow}_{m}\left(n_{1}, d_{1}\right) \times \cdots \times \operatorname{Chow}_{m}\left(n_{q}, d_{q}\right),
$$

parametrizing $(q+1)$-tuples $\left(X, D_{1}, \ldots, D_{q}\right)$ where $X$ is a smooth subvariety of dimension $n$ and degree $d$ in $\mathbb{P}^{m}$ and each $D_{i}$ is a smooth subvariety of dimension $n_{i}$ and degree $d_{i}$ contained in $X$.

In [Hwa05, Hwang generalizes the effective bounds on the number of components in Kol96] to the above defined Chow varieties. We will use his bound to derive an effective estimate on the number of toroidal compactifications.

The first thing to do is to bound the degree of each component of $D$ when embedded into some fixed projective space.

Lemma 3.16. Let $(X, D)$ be a toroidal compactification. Let $d_{i}$ be the degree of $D_{i}$ under the embedding given by Theorem 3.13. Then for any $i$

$$
d_{i} \leq\left(\frac{3}{2}\right)^{n}(n+1)^{3 n-3}\left(K_{X}+D\right)^{n} .
$$

Proof. Let $A$ be the very ample divisor obtained in the proof of Theorem 3.6 Recall that this divisor is of the form

$$
A=M\left(K_{X}+D\right)-\left(n^{3}+2 n+5\right) D
$$

for some positive integer $M$. Since $\left(n^{3}+2 n+5\right)<(n+1)^{3}$, we obtain that

$$
d_{i}=D_{i} \cdot A^{n-1} \leq(-1)^{n-1}(n+1)^{3 n-3} D^{n} \leq\left(\frac{3}{2}\right)^{n}(n+1)^{3 n-3}\left(K_{X}+D\right)^{n}
$$

where the last inequality is obtained thanks to Lemma 3.9 . 
Finally, we can prove an effective version of Wang's finiteness theorem.

Corollary 3.17. Fix two positive integers $n$ and $V$. Then the number of toroidal compactifications with $\operatorname{dim}(X)=n$ and $\left(K_{X}+D\right)^{n} \leq V$ is less than

$$
\sum_{q=1}^{q_{0}} d_{0}^{q+1}\left(\begin{array}{c}
(2 n+2) d_{0} \\
2 n+1
\end{array}\right)^{(2 n+2)(q+1)\left(\begin{array}{c}
2 n+1+d_{0} \\
2 n+1
\end{array}\right)},
$$

where $d_{0}=(2 n+2)^{3 n} V$ and $q_{0}=(3 / 2)^{n} V$.

Proof. Let $d$ be the degree of $X$ in $\mathbb{P}^{2 n+1}$ and let $d_{i}$ be the degree of $D_{i}$ for any $1 \leq i \leq q$. By definition, there exists a point in $\operatorname{Chow}_{2 n+1}(n, d ; n-1, \ldots, n-$ $\left.1 ; d_{1}, \ldots, d_{q}\right)$ parametrizing $(X, D)$. Theorem 4.1 in Fuj92 implies that $(X, D)$ is infinitesimally rigid under deformations of the pair. Therefore, the number of toroidal compactifications with fixed $\left(d, d_{1}, \ldots, d_{q}\right)$ is bounded by the number of components of the corresponding Chow variety. By Theorem 3.13, we can embed $X$ as a smooth subvariety of $\mathbb{P}^{2 n+1}$ with degree $d$ bounded by $(2 n+2)^{3 n} V$. Furthermore, each $D_{i}$ has degree $d_{i}$ bounded by $(3 / 2)^{n}(n+1)^{3 n-3} V$, by Lemma 3.16. Finally, for each $q>0$ there are at most $d_{0}^{q+1}$ choices of $\left(d, d_{1}, \ldots, d_{q}\right)$, which combined with Proposition 3.2 in [Hwa05] and Theorem 1.5, give the result.

Up to constants depending only on $n$, the above bound is of the form $V^{V^{2 n+2}}$.

3.4. Bounds on the Picard numbers. The first result of this section is a lower bound on the Picard number of a toroidal compactification $(X, D)$ in terms of the number of cusps of $X \backslash D$.

Theorem 3.18. Let $(X, D)$ be a toroidal compactification of dimension $n \geq 2$. Let $q$ be the number of components of $D$. Then $q<\rho(X)$, where $\rho(X)$ is the Picard number of $X$.

Proof. Suppose by contradiction that $\rho(X) \leq q$. Let $H$ be an ample divisor on $X$. Then there exists a non trivial relation

$$
a_{0} H+\sum_{i=1}^{q} a_{i} D_{i} \equiv 0
$$

where $a_{i} \in \mathbb{R}$. Since $D_{i} \cdot D_{j}^{n-1}=0$ for all $j \neq i$, the compactifying divisors are not numerically equivalent and we can assume $a_{0}=1$. Intersecting the above relation with a curve $C$ contained in $D_{i}$, we get that $a_{i}>0$ for all $i$. On the other hand

$$
0=H^{n-1} \cdot\left(H+\sum_{i=1}^{q} a_{i} D_{i}\right)=H^{n}+\sum_{i=1}^{q} a_{i} D_{i} \cdot H^{n-1}>0
$$

gives a contradiction.

We now bound from above the Picard number of a toroidal compactification in terms of its volume. Note that, because of Corollary 3.17 there are finitely many toroidal compactifications with given upper bound on the volume. As a result, there is a bound on their Picard numbers depending only on an upper bound on the volume. The next theorem shows how the techniques developed in this paper can be nicely applied to derive an explicit bound. 
Theorem 3.19. Let $(X, D)$ be a toroidal compactification of dimension $n \geq 3$. Then

$$
\rho(X) \leq 30+4\left(1+(n-2)\left(2 n^{2}+3\right)\right)^{2}\left(2 n^{2}+3\right)^{n-2} L^{n} .
$$

Proof. By Theorem 1.1. we know that $K_{X}+\alpha D$ is ample for any $\alpha \in\left(\frac{1}{3}, 1\right)$. In particular, $2 K_{X}+D$ is an ample $\mathbb{Z}$-divisor. By Anghern-Siu [AS95], we know that $K_{X}+\left(n^{2}+1\right)\left(2 K_{X}+D\right)$ is ample and base point free. Let us define

$$
L_{1}=K_{X}+\left(n^{2}+1\right)\left(2 K_{X}+D\right), \quad L_{2}=K_{X}+(n-2) L_{1} .
$$

Note that $L_{2}$ is ample. In fact, by Theorem 1.1 it suffices to observe that

$$
L_{2}=\left(1+(n-2)+2(n-2)\left(n^{2}+1\right)\right)\left(K_{X}+\frac{(n-2)\left(n^{2}+1\right)}{1+(n-2)+2(n-2)\left(n^{2}+1\right)} D\right)
$$

with

$$
\frac{(n-2)\left(n^{2}+1\right)}{1+(n-2)+2(n-2)\left(n^{2}+1\right)}>\frac{1}{3}
$$

for any $n \geq 3$. Since $L_{1}$ is base point free, by Bertini's theorem, see page 137 in GH78, the generic element in the linear system $\left|L_{1}\right|$ is smooth. Let us consider $(n-2)$ distinct generic elements in $\left|L_{1}\right|$, say $S_{1}, S_{2}, \ldots, S_{n-2}$, and let us denote by $S$ their intersection. $S$ is then a smooth surface in $X$. By reiterating $(n-2)$-times the adjunction formula, we obtain that

$$
L_{2}^{2} \cdot L_{1}^{n-2}=K_{S}^{2}
$$

where $K_{S}$ is ample since $L_{2}$ is ample over $X$. To compute the intersection number $L_{2}^{2} \cdot L_{1}^{n-2}$ it is convenient to express $L_{1}$ and $L_{2}$ in terms of the log-canonical divisor $L=K_{X}+D$. Thus, we have

$$
L_{1}=\left(2 n^{2}+3\right) L-\left(n^{2}+2\right) D,
$$

and

$$
L_{2}=\left(1+(n-2)\left(2 n^{2}+3\right)\right) L-\left(1+(n-2)\left(n^{2}+2\right)\right) D,
$$

which then implies

$$
L_{2}^{2} \cdot L_{1}^{n-2}=\left(1+(n-2)\left(2 n^{2}+3\right)\right)^{2}\left(2 n^{2}+3\right)^{n-2} L^{n} .
$$

By the Lefschetz hyperplane theorem, see page 157 in GH78, we know that for $n=3$ then $h^{1,1}(X)$ injects into $h^{1,1}(S)$, while for $n>3$ the restriction map gives an isomorphism between $h^{1,1}(X)$ and $h^{1,1}(S)$. To conclude the proof, we need an estimate on the Picard number of a surface with ample canonical divisor in terms of $c_{1}^{2}$. Because of Noether's inequality, see Theorem 3.1 in [BHPV04], we have

$$
h^{1,1}(S) \leq 30+4 K_{S}^{2}
$$

so that

$$
h^{1,1}(X) \leq 30+4\left(1+(n-2)\left(2 n^{2}+3\right)\right)^{2}\left(2 n^{2}+3\right)^{n-2} L^{n},
$$

which then gives an estimate of the Picard number of $X$ in terms of the top self intersection of $K_{X}+D$. 
For a two dimensional version of Theorem 3.19 and much more the interested reader may refer to $\mathrm{DiC13}$.

\section{REFERENCES}

[AS95] U. Angehrn and Y.-T Siu, Effective freeness and point separation for adjoint bundles, Invent. Math. 122 (1995), no. 2, 291-308.

[AMRT10] A. Ash, D. Mumford, M. Rapoport, Y.-S. Tai, Smooth compactifications of locally symmetric varieties. Second edition. Cambridge Mathematical Library. Cambridge University Press, Cambridge, 2010.

[BHPV04] W. P. Barth, K. Hulek, C. A. Peters, A. Van de Ven Compact complex surfaces, Ergebnisse der Mathematik und ihrer Grenzgebiete. 3. Folge. A Series of Modern Surveys in Mathematics, 4. Springer-Verlag, Berlin, 2004.

[BB66] W. L. Baily, A. Borel, Compactifications of arithmetic quotients of bounded symmetric domains, Ann. of Math., 84 (1966), no.2, 442-528.

[BJ06] A. Borel, L. Ji, Compactifications of locally symmetric spaces. Mathematics: Theory \& Applications, Birkäuser Boston, Inc. Boston, MA, 2006.

[DM93] P. Deligne, G. Mostow, Commensurabilities among lattices in PU(1,n), Annals of Mathematics Studies 132, Princeton University Press, Princeton, NJ, 1993.

[Dem92] J.-P. Demailly, Regularization of closed positive currents and intersection theory, $J$. Alg. Geom. 1 (1992), 361-409.

[DiC12] G. Di Cerbo, L. F. Di Cerbo, Positivity questions in Kähler-Einstein theory, arXiv:1210.0218 [mathDG], 2012.

[DiC13] G. Di Cerbo, L. F. Di Cerbo, A sharp cusp count for complex hyperbolic surfaces and related results, arXiv:1312.5368 [mathDG], 2013.

[Ebe96] P. Eberlein, Geometry of nonpositively curved manifolds, Chicago Lectures in Mathematics. University of Chicago Press, Chicago, IL, 1996.

[Fuj92] A. Fujiki, An $L^{2}$ Dolbeaut lemma and its applications, Publ. Res. Inst. Math. Sci. 28 (1992), 845-884.

[GH78] P. Griffiths, J. Harris, Principles of Algebraic Geometry. Pure and Applied Mathematics. Wiley-Interscience, New York, NY, 1978.

[Gro82] M. Gromov, Volume and bounded cohomology, Publ. Math. Inst. Hautes Etudes Sci. 56 (1982), 5-99.

[Har77] R. Hartshorne, Algebraic Geometry, Springer-Verlag, New York 1977, Graduate Texts In Mathematics, No. 52.

[Hei02] G. Heier, Effective freeness of adjoint line bundles, Doc. Math. 7 (2002), 31-42.

[Hir71] F. Hirzebruch, The Hilbert modular group, resolution of the singularities at the cusps and related problems. Séminarie Bourbaki, 23ème année (1970/1971), Exp. No. 396, pp. 275-288. Lecture Notes in Math., Vol. 244, Springer, Berlin, 1971.

[Hir84] F. Hirzebruch, Chern numbers of algebraic surfaces: an example. Math. Ann. 266 (1984), 351-356.

[Hum98] C. Hummel, Rank one lattices whose parabolic isometries have no rotation part. Proc. Am. Math. Soc. 126 (1998), 2453-2458.

[HS96] C. Hummel, V. Schroeder, Cusp closing in rank one symmetric spaces. Invent. Math. 123 (1996), 283-307.

[Hwa04] J.-M. Hwang, On the volumes of complex hyperbolic manifolds with cusps, Internat. J. Math. 15 (2004), no. 6, 567-572.

[Hwa05] J.-M. Hwang, On the number of complex hyperbolic manifolds of bounded volume, Internat. J. Math. 8 (2005), 863-873.

[JS93] S. Ji, B. Shiffman, Properties of compact complex manifolds carrying closed positive currents. J. Geom. Anal. 3 (1993), 37-61.

[Kaw97] Y. Kawamata, On Fujita's freeness conjecture for 3-folds and 4-folds, Math. Ann. 308 (1997), no. 3, 491-505. 
[Kol96] J. Kollár, Rational curves on algebraic varieties, Ergebnisse der Mathematik und ihrer Grenzgebiete. 3. Folge. A Series of Modern Surveys in Mathematics, vol. 32. Springer-Verlag, Berlin, 1996.

[Kol97] J. Kollár, Singularities of pairs, Algebraic Geometry, Santa Cruz 1995, Proc. Symp. Pure Math, vol. 62, Amer. Math. Soc., Providence, RI, 1997, 221-287.

[KM98] J. Kollár and S. Mori, Birational geometry of algebraic varieties, Cambridge Tracts in Mathematics, vol. 134, Cambridge University Press, 1998.

[Laz04a] R. Lazarsfeld, Positivity in algebraic geometry I, Ergebnisse der Mathematik und ihrer Grenzgebiete, vol. 48, Berlin: Springer 2004.

[Laz04b] R. Lazarsfeld, Positivity in algebraic geometry II, Ergebnisse der Mathematik und ihrer Grenzgebiete, vol. 49, Berlin: Springer 2004.

[Mar84] G. Margulis, Arithmeticity of the irreducible lattices in semisimple groups of rank gretaer than 1. Invent. Math. 76 (1984), 93-120.

[Mok12] N. Mok, Projective algebraicity of minimal compactifications of complex-hyperbolic space forms of finite-volume. Perspective in analysis, geometry, and topology, 331354, Prog. Math., 296, Birkhäuser/Springer, New York, 2012.

[Mor82] S. Mori, Threefolds whose canonical bundle is not numerically effective, Ann. of Math 116 (1982), 133-176.

[Mum77] D. Mumford, Hirzebruch's Proportionality Theorem in the Non-Compact Case. Invent. Math. 42 (1977), 239-272.

[Par98] J. R. Parker, On the volume of cusped, complex hyperbolic manifolds and orbifolds, Duke Math. J. 94 (1998), 433-464.

[Rei88] I. Reider, Vector bundles of rank 2 and linear systems on algebraic surfaces, Ann. Math. 127 (1988), 309-316.

[Sib85] N. Sibony, Quelques problemes de prolongement de courants en analyse complexe. Duke Math. J. 52 (1985), 157-197.

[SY82] Y. T. Siu, S. T. Yau, Compactification of negatively curved complete Kähler manifolds of finite volume, Seminars in Differential Geometry, pp. 363-380, Ann. of Math. Stud., Vol. 102, Princeton Univ. Press, Princeton, N. J., 1982.

[Tak93] S. Takayama, Ample vector bundles on open algebraic varieties. Publ. RIMS Kyoto Univ. 29 (1993), 885-910.

[Wis89] J. Wiśniewski, Length of extremal rays and generalized adjunction, Math. Z. 200 (1989), no. 3, 409-427.

[Wis91] J. Wiśniewski, On contractions of extremal rays of Fano manifolds, J. Reine Angew. Math. 417 (1991), 141-157.

[Wan72] H. C. Wang, Topics on totally discontinuous groups, Symmetric Spaces, 459-487, edited by W. B. Boothby and G. L. Weiss, Pure and Appl. Math. 8, Marcel Dekker, New York, 1972.

Department of Mathematics, Princeton University, Princeton NJ 08544-1000, USA

E-mail address: gdi@math.princeton.edu

Department of Mathematics, Duke University, Durham NC 27708-0320, USA

E-mail address: luca@math.duke.edu 